\title{
Rényi entropy of the U(3) vibron model
}

\author{
E. Romera \\ Departamento de Física Atómica, Molecular y Nuclear and Instituto Carlos I de Física Teórica y Computacional, \\ Universidad de Granada, Fuentenueva s/n, 18071 Granada, Spain \\ R. del Real \\ Departamento de Física Atómica, Molecular y Nuclear, \\ Universidad de Granada, Fuentenueva s/n, 18071 Granada, Spain \\ M. Calixto \\ Departamento de Matemática Aplicada, Universidad de Granada, Fuentenueva s/n, 18071 Granada, Spain \\ S. Nagy \\ Department of Theoretical Physics, University of Debrecen, H-4010 Debrecen, \\ Hungary and MTA-DE Research Group in Particle Physics, H-4010 Debrecen P.O.Box 105, Hungary
}

\author{
Á. Nagy \\ Department of Theoretical Physics, University of Debrecen, H-4010 Debrecen, Hungary
}

(Dated: September 7, 2012)

\begin{abstract}
Rényi entropies and variances are determined in the vibron model. They provide a sharp detector for the quantum (shape) phase transition (from linear to bent) at the critical value $\xi_{c}$ of a control parameter $\xi$. Numerical results are complemented and compared with a variational approximation in terms of parity-symmetry-adapted coherent (Schödinger's catlike) states, which provide a good approximation to describe delocalization properties of the ground state of vibron models across the critical point for $N$-size molecules.
\end{abstract}

PACS numbers: 05.30.Rt,33.15.Bh, 89.70.cf

\section{INTRODUCTION}

Recently, quantum phase transitions (QPT) [1] have received increasing attention. While in classical phase transitions it is a change in the temperature that induces an abrupt change in the physical properties of a system, quantum phase transitions take place at absolute zero of temperature. In these models, one finds different quantum phases connected to specific geometric configurations of the ground state and related to distinct dynamic symmetries of the Hamiltonian. The QPT occurs as a function of a control parameter $\xi$ that appears in the Hamiltonian $H$, for us in the form of a convex combination $H(\xi)=(1-\xi) H_{1}+\xi H_{2}$. At $\xi=0$ the system is in phase I, characterized by the dynamical symmetry $G_{1}$ of $H_{1}$, and at $\xi=1$ the system is in phase II, characterized by the dynamical symmetry $G_{2}$ of $H_{2}$. At some critical point $\xi_{c}$ there is an abrupt change in the symmetry and structure of the ground state wavefunction.

On the other hand, large correlations and delocalization are fundamental properties at the transition point and some information theoretical measures of delocalization has been recently studied to characterize QPT showing that they change significantly at the transition point [2-6]. In particular it has been seen that the Rényi entropy, relative complexity and Fisher-Rényi information product detect the quantum phase transition in the Dicke model [7-9]. Rényi entropy [6] of order $\alpha$ for a density function $\varrho$ normalized to one is defined by

$$
R^{\alpha} \equiv \frac{1}{1-\alpha} \ln \int \varrho^{\alpha}(q) d q, \quad \text { for } \quad 0<\alpha<\infty, \quad \alpha \neq 1 .
$$

This quantity is a one-parameter extension of Shannon entropy [10] as the Rényi entropy tends to the Shannon entropy

$$
S=-\int \varrho(q) \ln \varrho(q) d q
$$

when $\alpha \rightarrow 1$. Rényi entropy has been applied in several fields of quantum physics, such as quantum entanglement [11], quantum communication protocols [12], quantum correlations [13], localization properties [14], quantum revivals [15] or atomic physics [16-19]. These information measures sometimes give a description of the QPT of better quality than standard variance

$$
(\Delta q)^{2}=\left\langle q^{2}\right\rangle-\langle q\rangle^{2}=\int q^{2} \varrho(q) d q-\left(\int q \varrho(q) d q\right)^{2}
$$

which scales with the number of 'particles' and therefore proves to be divergent in the thermodynamic limit (see e.g. $[4,20]$ for the case of the Dicke model).

In this paper we will study Rényi entropy as a measure of delocalization in the context of the "vibron models" [21-23], interacting boson models which exhibit a second order shape phase transition from linear to bent. These 
models have been used to study the rovibrational properties in diatomic and polyatomic molecules and have turned to be very useful to study symmetry properties of quantum systems. They are suitable to investigate quantum phase transitions, too. Here, two dimensional (2D) vibron models are selected to demonstrate how the Rényi entropy reflects QPT. In section II the algebraic approach to 2D-vibron models has been briefly reviewed. We shall present ground state Rényi entropies as a measure of delocalization across the QPT point. Then we shall consider the variational description of the ground state with coherent states (CS) adapted to the parity symmetry of the system (introduced in [24]) showing that Rényi entropy of this variational approximation detects a sudden delocalization of the ground state wave packet across the critical point. Finally we shall present some analytical results related with the Rényi entropy of the parity-symmetry adapted coherent states.

\section{VIBRON MODEL}

2D-vibron models describe a system containing a dipole degree of freedom constrained to planar motion. Elementary excitations are (creation and annihilation) $2 \mathrm{D}$ vector $\tau$-bosons $\left\{\tau_{x}^{\dagger}, \tau_{y}^{\dagger}, \tau_{x}, \tau_{y}\right\}$ and a scalar $\sigma$-boson $\left\{\sigma^{\dagger}, \sigma\right\}$. It is convenient to introduce circular bosons: $\tau_{ \pm}=\mp\left(\tau_{x} \mp i \tau_{y}\right) / \sqrt{2}$. The nine generators of the $U(3)$ algebra are bilinear products of creation and annihilation operators, in particular:

$$
\begin{gathered}
\hat{n}=\tau_{+}^{\dagger} \tau_{+}+\tau_{-}^{\dagger} \tau_{-}, \hat{n}_{s}=\sigma^{\dagger} \sigma \\
\hat{l}=\tau_{+}^{\dagger} \tau_{+}-\tau_{-}^{\dagger} \tau_{-}, \\
\hat{D}_{+}=\sqrt{2}\left(\tau_{+}^{\dagger} \sigma-\sigma^{\dagger} \tau_{-}\right), \hat{D}_{-}=\sqrt{2}\left(-\tau_{-}^{\dagger} \sigma+\sigma^{\dagger} \tau_{+}\right),
\end{gathered}
$$

denote the number operator of vector $\hat{n}$ and scalar $\hat{n}_{s}$ bosons, 2D angular momentum $\hat{l}$ and dipole $\hat{D}_{ \pm}$operators, respectively (see [25] for the reminder four operators $\hat{Q}_{ \pm}, \hat{R}_{ \pm}$, which will not be used here). Assuming the total number of bosons $\hat{N}=\hat{n}+\hat{n}_{\sigma}$ and the 2D angular momentum $\hat{l}$ to be conserved, there are only two dynamical symmetry limits, $G_{1}=U(2)$ and $G_{2}=S O(3)$, associated with two algebraic chains starting from $U(3)$ and ending in $S O(2)$ : the so-called 'cylindrical' and 'displaced' oscillator chains. A general Hamiltonian of the $U(3)$ vibron model with only one- and two-body interactions can be expressed in terms of linear and quadratic Casimir operators of all the subalgebras contained in the dynamical symmetry algebra chains. To capture the essentials of the phase transition from the $G_{1}$-phase (linear) to the $G_{2}$-phase (bent) it is enough to consider a convex combination of the linear $C_{1}(U(2))=\hat{n}$ and quadratic $C_{2}(S O(3))=\hat{W}^{2}=\left(\hat{D}_{+} \hat{D}_{-}+\hat{D}_{-} \hat{D}_{+}\right) / 2+\hat{l}^{2}$ Casimir operators of the corresponding dynamical symmetries. In particular, we shall consider the essential Hamiltonian
$[25]$

$$
\hat{H}=(1-\xi) \hat{n}+\xi \frac{N(N+1)-\hat{W}^{2}}{N-1},
$$

where the (constant) quantum number $N$ is the total number of bound states that labels the totally symmetric $(N+1)(N+2) / 2$ dimensional representation $[N]$ of $U(3)$. It is known (see [25] and later on Sec. IIIB) that this model exhibits a (shape) QPT at $\xi_{c}=0.2$ and we shall see that Rényi entropies provide sharp indicators of this QPT.

The Hilbert space is spanned by the orthonormal basis vectors

$$
|N ; n, l\rangle=\frac{\left(\sigma^{\dagger}\right)^{N-n}\left(\tau_{+}^{\dagger}\right)^{\frac{n+l}{2}}\left(\tau_{-}^{\dagger}\right)^{\frac{n-l}{2}}}{\sqrt{(N-n) !\left(\frac{n+l}{2}\right) !\left(\frac{n-l}{2}\right) !}}|0\rangle,
$$

where the bending quantum number $n=N, N-1, N-$ $2, \ldots, 0$ and the angular momentum $l= \pm n, \pm(n-$ $2), \ldots, \pm 1$ or 0 ( $n=$ odd or even $)$ are the eigenvalues of $\hat{n}$ and $\hat{l}$, respectively. The matrix elements of $\hat{W}^{2}$ can be easily derived (see e.g. [25]):

$$
\begin{aligned}
& \left\langle N ; n^{\prime}, l\left|\hat{W}^{2}\right| N ; n, l\right\rangle= \\
& \left((N-n)(n+2)+(N-n+1) n+l^{2}\right) \delta_{n^{\prime}, n} \\
& -((N-n+2)(N-n+1)(n+l)(n-l))^{\frac{1}{2}} \delta_{n^{\prime}, n-2} \\
& -((N-n)(N-n-1)(n+l+2)(n-l+2))^{\frac{1}{2}} \delta_{n^{\prime}, n+2} .
\end{aligned}
$$

From these matrix elements, it is easy to see that time evolution preserves the parity $e^{i \pi n}$ of a given state $|N, n, l\rangle$. That is, the parity operator $\hat{\Pi}=e^{i \pi \hat{n}}$ commutes with $\hat{H}$ and both operators can then be jointly diagonalized. We shall take this fact into account when proposing parity-symmetry-adapted ansäte in subsection III B.

In order to compute information measures, it will be useful to write the basis wave functions (6) in 'position' $q_{i}=\frac{1}{\sqrt{2}}\left(a_{i}^{\dagger}+a_{i}\right)$ representation, with $\left(a_{0}, a_{1}, a_{2}\right) \equiv$ $\left(\sigma, \tau_{+}, \tau_{-}\right)$our three oscillator operators, which can be written in terms of Hermite polynomials $H_{k}(x)$ as:

$$
\begin{aligned}
\langle q \mid N ; n, l\rangle= & \frac{2^{-N / 2} \pi^{-3 / 4} e^{-\left(q_{0}^{2}+q_{1}^{2}+q_{2}^{2}\right) / 2}}{\sqrt{(N-n) !\left(\frac{n+l}{2}\right) !\left(\frac{n-l}{2}\right) !}} \\
& \times H_{N-n}\left(q_{0}\right) H_{\frac{n+l}{2}}\left(q_{1}\right) H_{\frac{n-l}{2}}\left(q_{2}\right) .
\end{aligned}
$$

\section{RÉNYI ENTROPY AND VARIANCES}

\section{A. Numerical Study}

Let us denote by

$$
\left|\psi_{\xi}^{(N)}\right\rangle=\sum_{n=0}^{N} \sum_{m=0}^{n} c_{n, m}^{(N)}(\xi)|N ; n, l=n-2 m\rangle
$$


the exact ground state of our system obtained by diagonalization of the Hamiltonian (5) in terms of the basis vectors $(6)$ with coefficients $c_{n, m}^{(N)}(\xi)$ (which have to be calculated numerically). Let us denote by $\psi_{\xi}^{(N)}(q)=$ $\left\langle q \mid \psi_{\xi}^{(N)}\right\rangle$ the corresponding wave function in position representation $q=\left(q_{0}, q_{1}, q_{2}\right)$ and by $\rho_{\xi}^{(N)}(q)=\left|\psi_{\xi}^{(N)}(q)\right|^{2}$ the ground state density distribution, for which the Rényi entropy

$$
R_{N}^{\alpha}(\xi)=\frac{1}{1-\alpha} \ln \int_{\mathbb{R}^{3}}\left(\rho_{\xi}^{(N)}(q)\right)^{\alpha} d^{3} q
$$

with $\alpha>0$, is a function of the control parameter $\xi$ for each $N$.

In Fig. 1 we represent Rényi (and Shannon) entropies of the ground state of the vibron model in position representation for a few selected values of $\alpha=1,2,3 / 4$ and $N=8,16,20$, as a function of $\xi$. We can see that Rényi entropies undergo a sudden growth across the phase transition critical point $\xi_{c} \simeq 0.2$, thus indicating a delocalization of the ground state wave packet in the second (bent) phase, $\xi>0.2$. Moreover, it can be seen in figure 1 that $R_{N}^{\alpha}(\xi)$ is an increasing function of $N$ and $\xi$ and a decreasing function of $\alpha$.

One can verify that, for this model, the ground state wave function in momentum space $\tilde{\psi}_{\xi}^{(N)}(p)=\left\langle p \mid \psi_{\xi}^{(N)}\right\rangle$ (i.e., the Fourier transform of $\left.\psi_{\xi}^{(N)}(q)\right)$ has the same functional form as $\psi_{\xi}^{(N)}(q)$. Thus, we shall restrict ourselves to position space. Moreover, the mean values

$$
\left\langle q_{i}\right\rangle=\int_{\mathbb{R}^{3}} q_{i} \rho_{\xi}^{(N)}(q) d^{3} q=0, \quad i=0,1,2,
$$

are zero, so that standard uncertainty variance products will be given by the mean squares

$$
\left(\Delta q_{i}\right)^{2}=\left\langle q_{i}^{2}\right\rangle=\int_{\mathbb{R}^{3}} q_{i}^{2} \rho_{\xi}^{(N)}(q) d^{3} q, \quad i=0,1,2 .
$$

Rotational symmetry implies $\left\langle q_{1}^{2}\right\rangle=\left\langle q_{2}^{2}\right\rangle$. In Figure 2 we see that uncertainty in $q_{0}$ decreases, whereas uncertainty in $q_{1,2}$ increases, across the phase transition, and both quantities scale with $N$ (the size of the system). Moreover, the total variance:

$$
\left\langle q_{0}^{2}+q_{1}^{2}+q_{2}^{2}\right\rangle=\frac{3}{2}+N
$$

is constant (independent of $\xi$ ). This fact is related to the conservation of the total number of bosons $\hat{N}=a_{0}^{\dagger} a_{0}+$ $a_{1}^{\dagger} a_{1}+a_{2}^{\dagger} a_{2}$

\section{B. Variational Study}

The classical, thermodynamic or mean-field $N \rightarrow \infty$ limit of these models is studied by using an algorithm
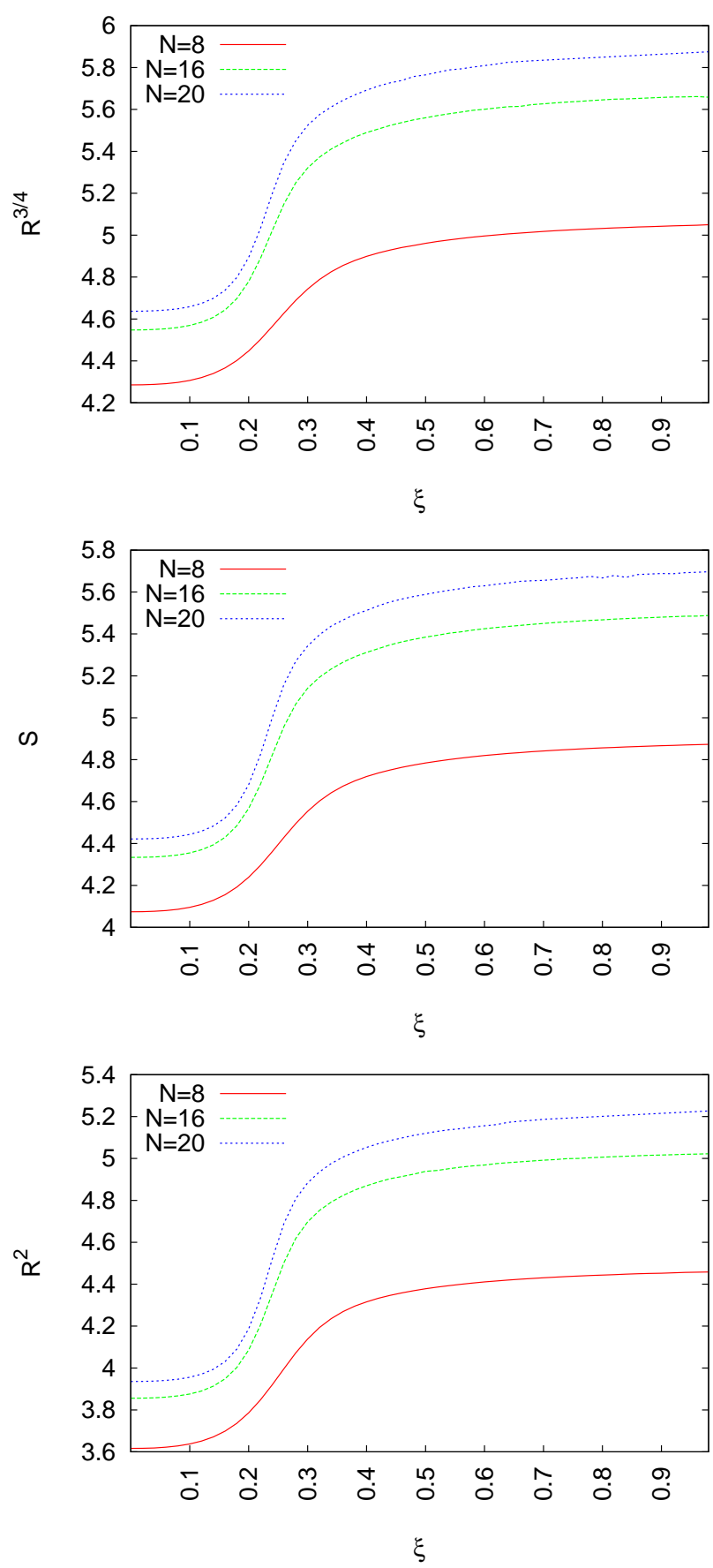

FIG. 1. (Color online) Rényi entropies $R_{N}^{\alpha}(\xi)$ for the exact (numerical) ground state of the vibron model as a function of $\xi$ for $\alpha=3 / 4$ (top panel), $\alpha=1$ (middle panel) and $\alpha=2$ (bottom panel) for $N=8,16$ and 20 .

introduced by Gilmore [26] which makes use of semiclassical (boson-condensate) Coherent States (CSs) (see e.g. [27-29] for standard references on CSs), as variational states to approximate the ground state energy, in particular 'projective' [25] CSs

$$
|N ; r\rangle \equiv \frac{1}{\sqrt{N !}}\left(b_{c}^{\dagger}\right)^{N}|0\rangle, \quad b_{c}^{\dagger}=\frac{1}{\sqrt{1+r^{2}}}\left(\sigma^{\dagger}+r \tau_{x}^{\dagger}\right),
$$



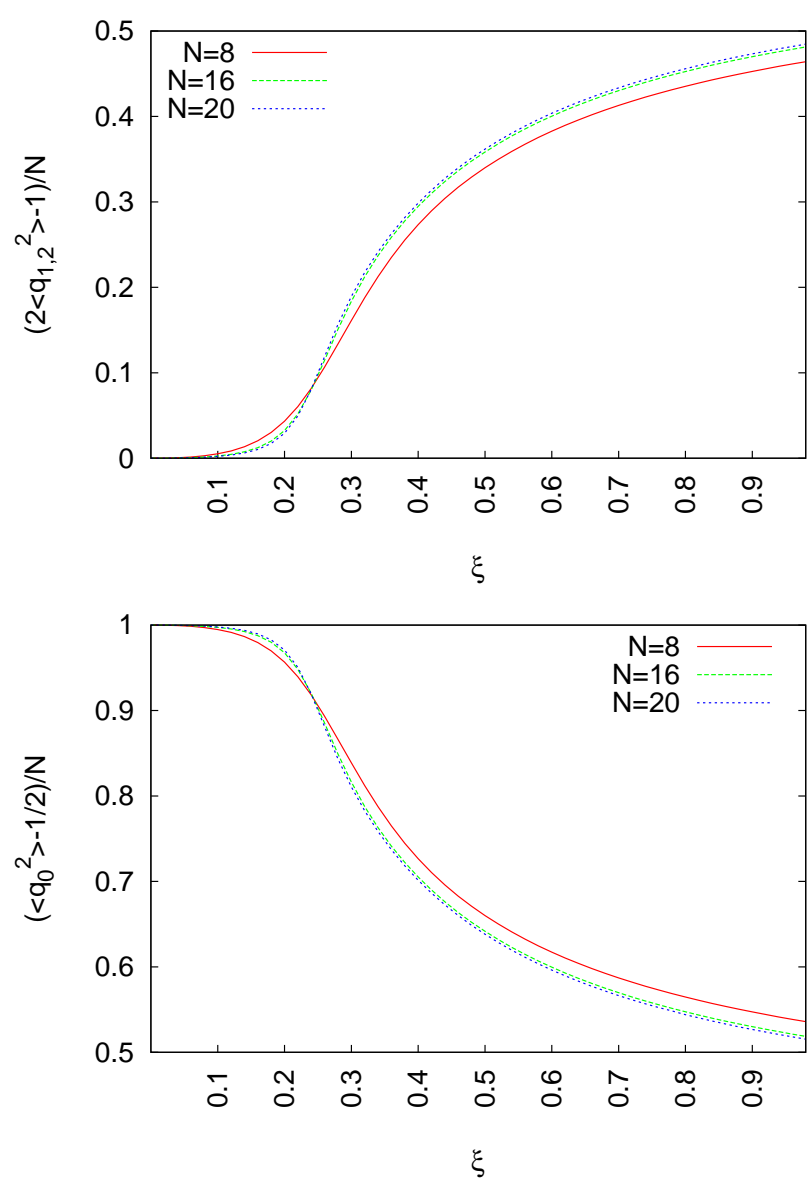

FIG. 2. (Color online) Rescaled variances $\left(2\left\langle q_{1,2}^{2}\right\rangle-1\right) / N$ (top panel) and $\left(\left\langle q_{0}^{2}\right\rangle-1 / 2\right) / N$ (bottom panel) for the exact (numerical) ground state of the vibron model as a function of $\xi$ for $N=8,16$ and 20 .

with $r \geq 0$ a free variational parameter and $b_{c}^{\dagger}$ the boson condensate. Other rotationally equivalent possibilities can be also considered [30]; moreover, intrinsic excitations can also be constructed by replacing the intrinsic boson $b_{c}$ with orthogonal excitation bosons, thus defining multi-species CSs (see e.g $[31,32]$ ). In this article we shall restrict ourselves to ground state ansäte. In position representation, this trial state can be written as:

$$
\begin{aligned}
\psi_{r}^{(N)}(q) & =\langle q \mid N ; r\rangle \\
& =\frac{e^{-\frac{1}{2}\left(q_{0}^{2}+q_{1}^{2}+q_{2}^{2}\right)} H_{N}\left(\frac{q_{0}-\frac{r}{\sqrt{2}}\left(q_{1}-q_{2}\right)}{\sqrt{1+r^{2}}}\right)}{2^{N / 2} \pi^{3 / 4} \sqrt{N !}},
\end{aligned}
$$

where we have used (7) at some stage.

The variational parameter $r$ is fixed by minimizing the ground state energy functional 'per particle' [25]:

$$
\begin{aligned}
\mathcal{E}_{\xi}(r) & =\frac{\langle\hat{H}\rangle}{N}=(1-\xi) \frac{\langle\hat{n}\rangle}{N}+\xi \frac{N(N+1)-\left\langle\hat{W}^{2}\right\rangle}{N(N-1)} \\
& =(1-\xi) \frac{r^{2}}{1+r^{2}}+\xi\left(\frac{1-r^{2}}{1+r^{2}}\right)^{2}
\end{aligned}
$$

where we have used $\langle\cdot\rangle$ as a shorthand for expectation values in $|N ; r\rangle$. From $\partial \mathcal{E}_{\xi}(r) / \partial r=0$ one gets the 'equilibrium radius' $r_{e}$ and the ground state energy $\mathcal{E}_{\xi}$ as a function of the control parameter $\xi$ :

$$
\begin{aligned}
& r_{e}(\xi)= \begin{cases}0, & \xi \leq \xi_{c}=1 / 5 \\
\sqrt{\frac{5 \xi-1}{3 \xi+1}}, & \xi>\xi_{c}=1 / 5\end{cases} \\
& \mathcal{E}_{\xi}\left(r_{e}(\xi)\right)= \begin{cases}\xi, & \xi \leq \xi_{c}=1 / 5 \\
\frac{-9 \xi^{2}+10 \xi-1}{16 \xi}, & \xi>\xi_{c}=1 / 5 .\end{cases}
\end{aligned}
$$

Then one finds that $d^{2} \mathcal{E}_{\xi}\left(r_{e}(\xi)\right) / d \xi^{2}$ is discontinuous at $\xi_{c}=1 / 5$ and the phase transition is said to be of second order.

Although $\left|N ; r_{e}(\xi)\right\rangle$ properly describes some meanfield quantities (namely, the mean energy) and groundstate behavior in the thermodynamic limit $N \rightarrow \infty$, it has been recently noticed in [24] that it does not capture the correct behavior for other ground state properties sensitive to the parity symmetry $\hat{\Pi}$ of the Hamiltonian like, for instance, vibration-rotation entanglement. Indeed, a far better variational description of the ground state is given in terms of the even-parity projected 'projective' CSs [24]:

$$
\psi_{r,+}^{(N)}(q) \equiv \frac{\psi_{r}^{(N)}(q)+\psi_{-r}^{(N)}(q)}{\sqrt{2\left(1+\left(\frac{1-r^{2}}{1+r^{2}}\right)^{N}\right)}} .
$$

In [24] we proved that (17) is a superposition of two non-overlapping (distinguishable) quasi-classical (coherent) wave packets, which justifies the term 'Schrödinger catlike' for these states. Symmetry adapted CSs have also been proposed in $[33,34]$ to study the Dicke model QPT.

The variational parameter $r$ in (17) is again computed by minimizing the ground state energy functional "per particle' $\mathcal{E}_{\xi,+}^{(N)}(r)=\langle\hat{H}\rangle_{+} / N$ as in (15), but now for the symmetric configuration (17), given in terms of the new mean values:

$$
\begin{aligned}
\frac{\langle\hat{n}\rangle_{+}}{N} & =\frac{r^{2}\left(\left(1+r^{2}\right)^{N-1}-\left(1-r^{2}\right)^{N-1}\right.}{\left(1+r^{2}\right)^{N}+\left(1-r^{2}\right)^{N}} \\
\frac{\left\langle\hat{W}^{2}\right\rangle_{+}}{N} & =2 \frac{\left(1+r^{2}\right)^{N}+\left(1-r^{2}\right)^{N-2}\left(1+2 N r^{2}+r^{4}\right)}{\left(1+r^{2}\right)^{N}+\left(1-r^{2}\right)^{N}} .
\end{aligned}
$$

Unlike $\mathcal{E}_{\xi}(r)$, the new energy functional $\mathcal{E}_{\xi,+}^{(N)}(r)$ depends on $N$. From $\partial \mathcal{E}_{\xi,+}^{(N)}(r) / \partial r=0$ we can obtain the new equilibrium radius $r_{e}^{(N)}(\xi)$. Figure 3 compares $r_{e}(\xi)$ in (16) with $r_{e}^{(N)}(\xi)$ for $N=8,16,60$. We observe that, in the thermodynamic limit, $r_{e}^{(\infty)}(\xi)=r_{e}(\xi)$. Figure 4 also compares the exact ground state energy density (as a function of $\xi$ ) with the 'cat' (17) $\mathcal{E}_{\xi,+}^{(N)}\left(r_{e}^{(N)}(\xi)\right)$ and 'mean-field' (14) $\mathcal{E}_{\xi}\left(r_{e}(\xi)\right)$ mean energy per particle. We see that the cat state (17) provides a lower energy value 


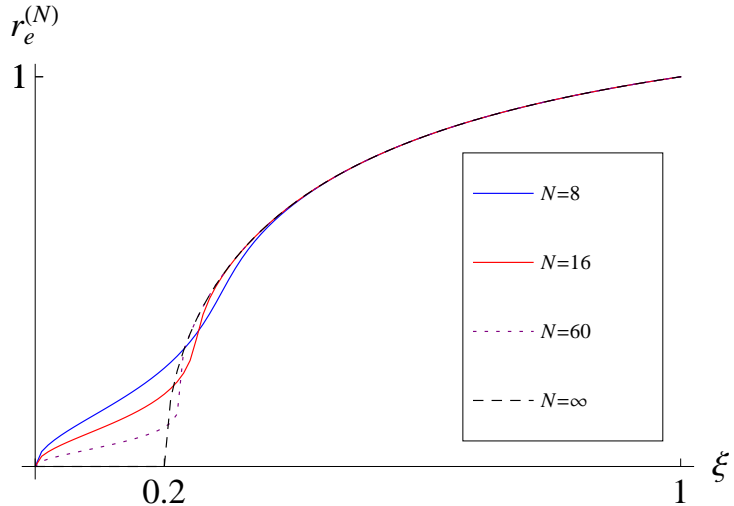

FIG. 3. (Color online) Equilibrium radius $r_{e}^{(N)}(\xi)$ of the cat approximation for $N=8,16$ and 60 . We are identifying $r_{e}^{(\infty)}(\xi)=r_{e}(\xi)$ (dashed), the equilibrium radius for the mean-field approximation.

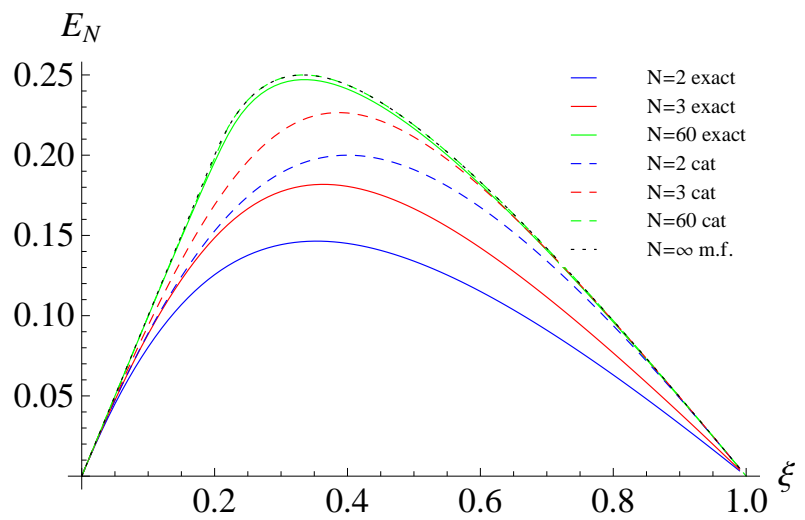

FIG. 4. (Color online) Exact ground state energy density (solid) against the 'cat mean energy per particle' $E_{N}^{+}(\xi) \equiv$ $\mathcal{E}_{\xi,+}^{(N)}\left(r_{e}^{(N)}(\xi)\right)$ (dashed) for $N=2, N=3$ and $N=60$. We are identifying $\mathcal{E}_{\xi,+}^{(\infty)}\left(r_{e}^{(\infty)}(\xi)\right)=\mathcal{E}_{\xi}\left(r_{e}(\xi)\right)$ (dotted), the mean energy per particle of the mean-field approximation. $E_{N}$ grows with $N$.

than the mean-field state (14) and tends to it in the thermodynamic limit $N \rightarrow \infty$, just as the exact ground energy density does. We have represented the two more unfavorable cases $N=2$ and 3. For $N>60$, the energy density values provided by exact, cat and mean-field configurations are already quite similar.

Let us denote by

$$
\begin{aligned}
\rho_{\xi}^{(N)}(q)_{\text {m.f. }} & =\left|\psi_{r_{e}(\xi)}^{(N)}(q)\right|^{2}, \\
\rho_{\xi}^{(N)}(q)_{\text {cat }} & =\left|\psi_{r_{e}^{(N)}(\xi),+}^{(N)}(q)\right|^{2},
\end{aligned}
$$

the variational ground state probability densities for the mean field (14) and cat (17) configurations. Figure 5 represents Rényi entropies $R_{N}^{\alpha}(\xi)$ for the variational ground state, 'mean-field' and 'cat' configurations as a function of $\xi$ for different values of $\alpha$ and $N$. We see that, whereas the Rényi entropy of the mean field configuration remains
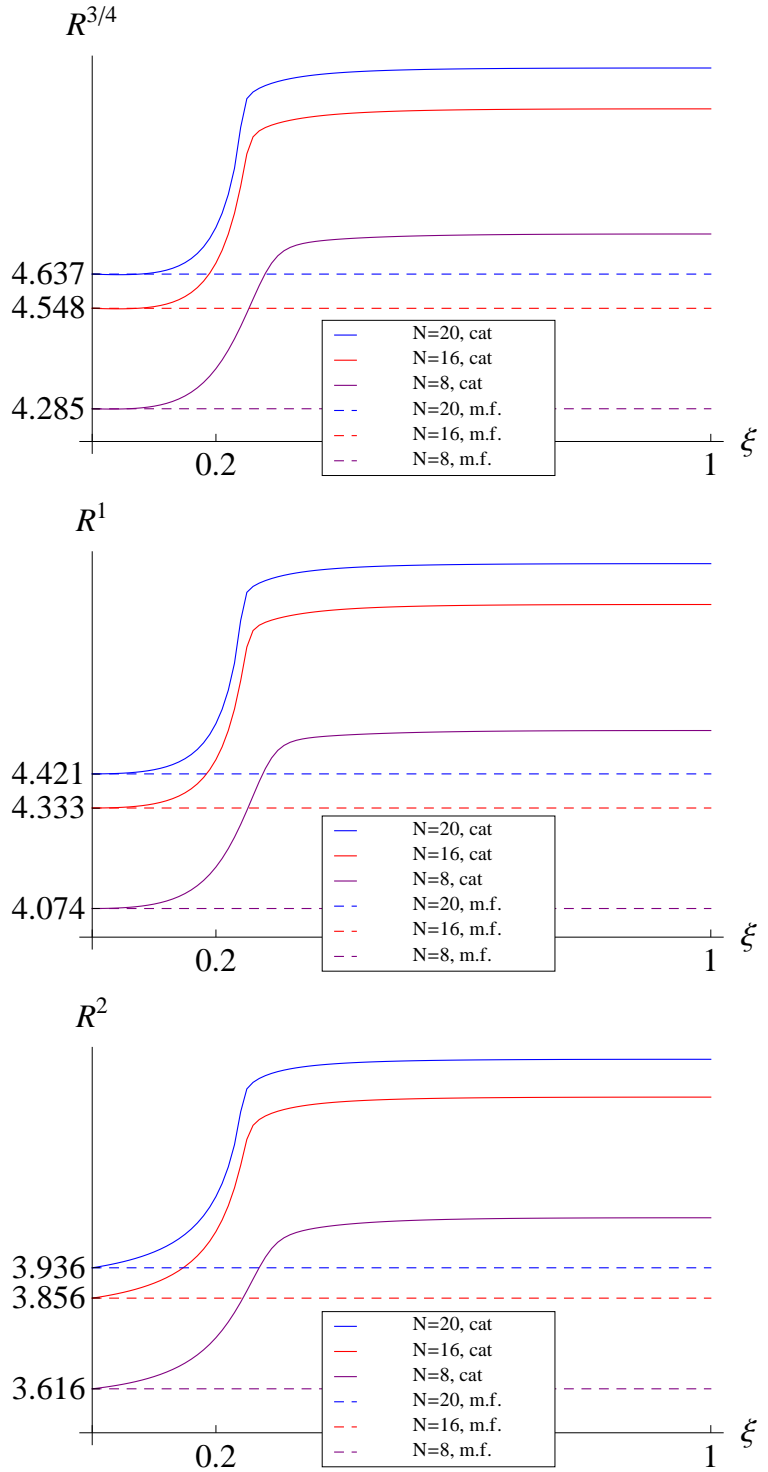

FIG. 5. (Color online) Rényi entropies $R_{N}^{\alpha}(\xi)$ for the variational ground state, 'mean-field' (constant dashed) and 'cat', configurations as a function of $\xi$ for $\alpha=3 / 4$ (top panel), $\alpha=1$ (middle panel) and $\alpha=2$ (bottom panel) for $N=8$, 16 and 20 .

constant with the control parameter $\xi$, the cat configuration captures the QPT at the critical point $\xi_{c}=0.2$, showing an entropy excess of $\Delta R_{N}^{\alpha}(\xi) \simeq 0.5$ between the both phases, thus accounting for the sudden delocalization of the ground state across the phase transition.

The values of $R_{N}^{\alpha}(\xi)$ for the variational cat state exactly coincide with the exact (numerical) values in the rigidly linear phase $\xi \simeq 0$, although they differ in the rigidly bent phase $\xi \simeq 1$. We can see in figure 6 together the exact (solid line), cat (dashed line) and mean-field (constant line) entropies, for $R_{20}^{\alpha}(\xi)$ with $\alpha=3 / 4,1$ and 2 (from top to bottom). Figure 6 shows that the numerical and cat entropies capture the delocalization while 


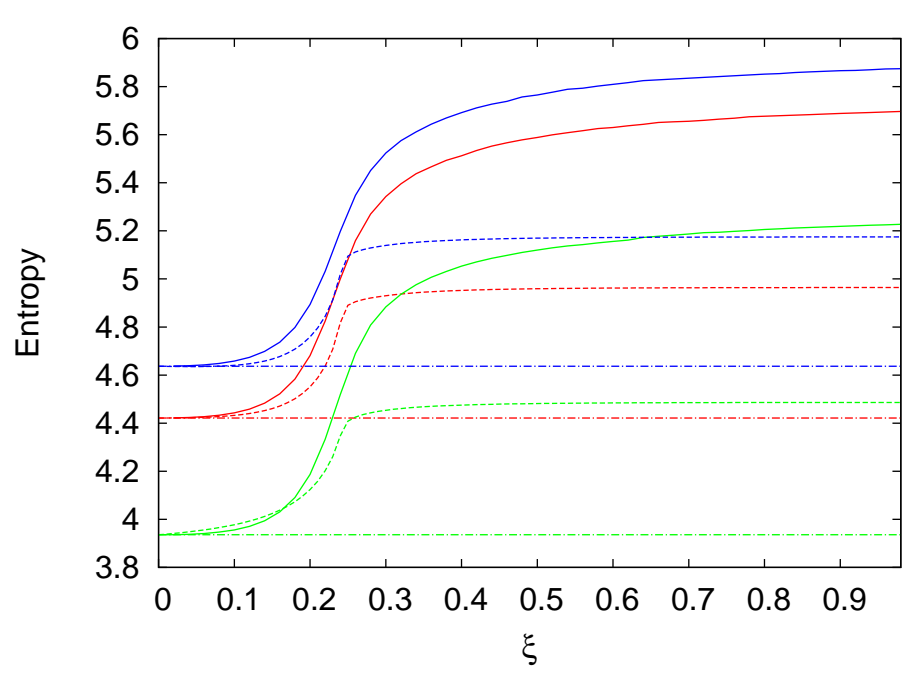

FIG. 6. (Color online) Rényi entropies $R_{N}^{\alpha}(\xi)$ for (from top to bottom) $\alpha=3 / 4,1$ and 2 , for the numerical (solid line), 'cat' (dots) and 'mean-field' (constant line) variational approximations as a function of $\xi$ for $N=20$.

the mean-field entropies remain constant. One can easily realize why the Rényi entropies (9) are constant for the mean field probability density (19). Actually, the change of variables $q^{\prime}=q \mathcal{R}(r)$, with $q=\left(q_{0}, q_{1}, q_{2}\right)$ and

$$
\mathcal{R}(r)=\frac{1}{\sqrt{1+r^{2}}}\left(\begin{array}{ccc}
1 & 0 & r \\
-\frac{r}{\sqrt{2}} & \frac{\sqrt{1+r^{2}}}{\sqrt{2}} & \frac{1}{\sqrt{2}} \\
\frac{r}{\sqrt{2}} & \frac{\sqrt{1+r^{2}}}{\sqrt{2}} & -\frac{1}{\sqrt{2}}
\end{array}\right)
$$

a (not unique) orthogonal matrix $\left(\mathcal{R} \mathcal{R}^{T}=1\right)$, allows us to write the mean field wave function (14) simply as

$$
\psi_{r}^{(N)}(q)=\frac{e^{-\frac{1}{2} q^{\prime 2}} H_{N}\left(q_{0}^{\prime}\right)}{2^{N / 2} \pi^{3 / 4} \sqrt{N !}},
$$

where we have used that $q^{2}=q^{2}$ (orthogonal transformation). Taking into account that the Lebesgue measure $d^{3} q$ in (9) is invariant under rotations $\left(d^{3} q=d^{3} q^{\prime}\right)$, and that the explicit dependence of $\psi_{r}^{(N)}(q)$ on $r$ disappears when written in terms of $q^{\prime}$, we finally get the independence of $R_{N}^{\alpha}$ on $\xi$ for the mean field probability density (19) (though the dependence of $r=r_{e}(\xi)$ on $\xi$ ).

At this point we have to underline that the cat approximation gives a good quantitative description in the rigidly linear phase, but it has a different value in the rigidly bent phase, although it qualitatively captures the exact behavior. We want to stress that one should expect some differences because the minimization of the Hamiltonian with these type of variational wave functions are known to provide an approximation to the exact ground state energy in order $1 / N$ (see e.g. figure 4 and $[35,36]$ for a deep study of this aspect of the problem. Integrals are numerically calculated for specific values of $\alpha, N$ and $\xi$. However, we can still provide an asymptotic $N \gg 1$ value of $R_{N}^{\alpha}(0)$ for some values of $\alpha$. In fact, taking into account that $r_{e}^{(N)}(0)=0, \forall N$, the cat state $(17)$ at $\xi=0$ factorizes as:

$$
\psi_{0,+}^{(N)}(q)=\frac{e^{-\frac{1}{2}\left(q_{0}^{2}+q_{1}^{2}+q_{2}^{2}\right)} H_{N}\left(q_{0}\right)}{2^{N / 2} \pi^{3 / 4} \sqrt{N !}} .
$$

Thus we can define the density at $\xi=0$ as the product of "rotational" times "vibrational" contributions

$$
\rho^{(N)}(q)=\left|\psi_{0,+}^{(N)}(q)\right|^{2}=\rho_{R}^{(N)}\left(q_{0}\right) \rho_{V}^{(N)}\left(q_{1}, q_{2}\right)
$$

with

$$
\begin{aligned}
\rho_{R}^{(N)}\left(q_{1}, q_{2}\right) & =\frac{e^{-\left(q_{0}^{2}+q_{1}^{2}\right)}}{\pi}, \\
\rho_{V}^{(N)}\left(q_{0}\right) & =\frac{e^{-q_{0}^{2}} H_{N}^{2}\left(q_{0}\right)}{2^{N} \sqrt{\pi} N !} .
\end{aligned}
$$

Rényi entropy at $\xi=0$ can be then written as the sum of two (rotational plus vibrational) contributions

$$
R_{N}^{\alpha}(0)=\tilde{R}_{N}^{\alpha}+V_{N}^{\alpha}
$$

with

$$
\begin{aligned}
\tilde{R}_{N}^{\alpha} & =\ln \pi-\frac{\ln \alpha}{1-\alpha} \\
V_{N}^{\alpha} & =\frac{1}{1-\alpha} \ln \left(\int \rho_{V}^{(N)}\left(q_{0}\right) d q_{0}\right) .
\end{aligned}
$$

The vibrational Rényi entropy takes the form

$$
\begin{gathered}
V^{\alpha}=\frac{1}{1-\alpha} \\
{\left[\ln \left(\int_{-\infty}^{\infty} e^{-\alpha q_{0}^{2}} H_{N}^{2 \alpha}\left(q_{0}\right) d q_{0}\right)-\alpha \ln \left(2^{N} \sqrt{\pi} N !\right)\right] .}
\end{gathered}
$$

In the limit $\alpha \rightarrow 1$, the vibrational Shannon entropy is obtained (it has already been determined by Assche et al. [37]):

$$
\begin{array}{r}
V_{N}^{1}=\ln \left(\sqrt{\pi} 2^{N} N !\right)+ \\
N+\frac{1}{2}+\frac{1}{2^{N} \sqrt{\pi} N !} S\left(H_{N}\right),
\end{array}
$$

where

$$
S\left(H_{N}\right)=-\int e^{-q_{0}^{2}} H_{N}^{2}\left(q_{0}\right) \ln \left(H_{N}\left(q_{0}\right)\right)^{2} d q_{0}
$$

is the vibrational Shannon entropy of the Hermite polynomials $H_{N}$. The latter has not been determined yet. For large $N$, however, the integral in Eq. (30) can be found $[38-42]$

$$
S\left(H_{N}\right) \approx N+\frac{3}{2}-\ln \pi-\ln \sqrt{2 N}, \quad N \gg 1,
$$

and the Shannon entropy has the asymptotic value

$$
V_{N}^{1} \approx \ln \frac{\pi \sqrt{2 N}}{e}, \quad N \gg 1 .
$$




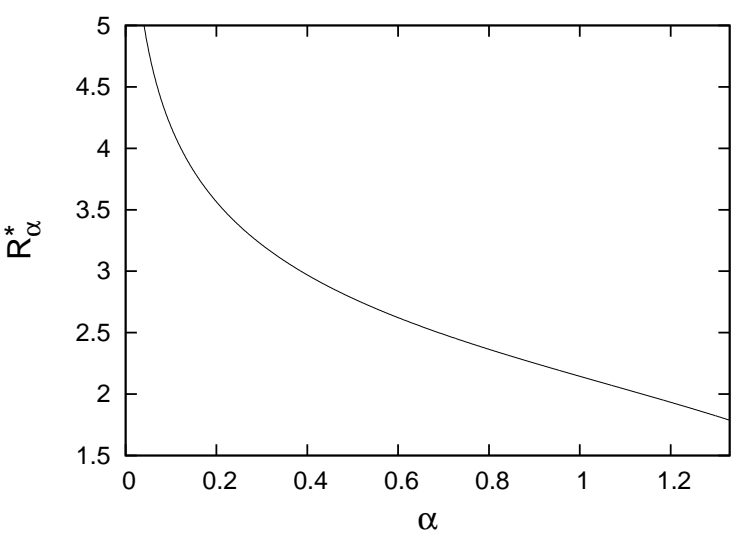

FIG. 7. The $\alpha$-dependent part $R_{\alpha}^{*}$ of the Rényi entropy for $\xi=0$.

No analytical expression of $V_{N}^{\alpha}$ has been found for general $\alpha$. However, we must mention that there also exist analytical results for the asymptotic case $N \gg 1$ for $\alpha \in(0,4 / 3][38,43]$ :

$$
\begin{array}{r}
V_{N}^{\alpha} \simeq \frac{1}{2} \ln (2 N+1)+\frac{1}{1-\alpha} \\
{\left[\alpha \ln \left(\frac{2}{\pi}\right)+\ln \left(\frac{\Gamma(\alpha+1 / 2) \Gamma(1-\alpha / 2)}{\Gamma(\alpha+1) \Gamma((3-\alpha) / 2)}\right)\right] .}
\end{array}
$$

The Shannon entropy calculated with this asymptotic expression is $S=R^{1}=4.13$ for $N=20$, while the numerical and variational results give 4.42 (see Fig. 5). The asymptotic expression for $R_{20}^{3 / 4}(0)$ gives 4.42 whereas the numerical and variational values are 4.37. To gain an insight into the $\alpha$-dependence of the Rényi entropy first note that in this case $(\xi=0)$ the $N$ and $\alpha$ dependence can be separated in the total Rényi entropy as

$$
R_{N}^{\alpha}(0)=V_{N}^{1}+R_{\alpha}^{*}
$$

$V_{N}^{1}$ is the asymptotic value for the Shannon vibrational entropy given by Eq. (32) and

$$
\begin{aligned}
R_{\alpha}^{*} \simeq & \frac{1}{1-\alpha} \ln \left[\frac{1}{\alpha}\left(\frac{2}{\pi}\right)^{\alpha} \frac{\Gamma(\alpha+1 / 2) \Gamma(1-\alpha / 2)}{\Gamma(\alpha+1) \Gamma((3-\alpha) / 2)}\right] \\
& +1
\end{aligned}
$$

includes the $\alpha$-dependence. Fig. 7 . presents $R_{\alpha}^{*}$ for $(0,4 / 3]$. We can see that $V_{N}^{1}$ and $R_{\alpha}^{*}$ are about the same order in magnitude for $N=20$. As $V_{N}^{1}$ contains the logarithm of $N$, it increases with $N$ rather slowly and $R_{\alpha}^{*}$ can be neglected only for very large $N$ (for instance, for $N=10^{23}$ we obtain $V_{N}^{1}=26.97$ ). The Rényi entropy behaves as $\frac{1}{2} \ln N$ for large $N$ in the interval $\alpha \in(0,4 / 3]$. We are tempted to conjecture the same behavior for other values of $\alpha$ and a similar behavior for other values of $\xi$.

Finally we compare variances for symmetric (cat) and non-symmetric (mean field) configurations. Let us simply denote by:

$$
\left\langle q_{i}^{2}\right\rangle_{+}(\xi)=\int_{\mathbb{R}^{3}} q_{i}^{2} \rho_{\xi}^{(N)}(q)_{\mathrm{cat}} d^{3} q, \quad i=0,1,2,
$$

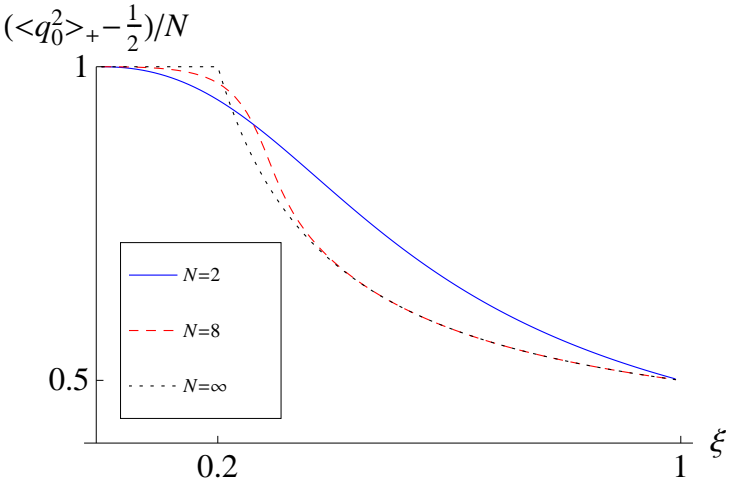

FIG. 8. (Color online) Normalized variance for the 'scalar' component $q_{0}$ in the cat $(N=2$ and 8$)$ and mean-filed $(N=$ $\infty)$ approximations.

the expectation values of squared position for symmetric (and $\left\langle q_{i}^{2}\right\rangle$ for non-symmetric) configurations. It is easy to realize that $\left\langle q_{i}\right\rangle_{+}=0=\left\langle q_{i}\right\rangle, \forall i$. Thus, fluctuations are given in terms of the mean squares:

$$
\begin{aligned}
& \left\langle q_{0}^{2}\right\rangle\left(r^{2}\right)=\frac{1}{2}+N \frac{1}{1+r^{2}}, \\
& \left\langle q_{1,2}^{2}\right\rangle\left(r^{2}\right)=\frac{1}{2}+\frac{N}{2} \frac{r^{2}}{1+r^{2}},
\end{aligned}
$$

for the non-symmetric configuration and

$$
\begin{aligned}
& \left\langle q_{0}^{2}\right\rangle_{+}\left(r^{2}\right)=\frac{\left(1+r^{2}\right)^{N}\left\langle q_{0}^{2}\right\rangle\left(r^{2}\right)+\left(1-r^{2}\right)^{N}\left\langle q_{0}^{2}\right\rangle\left(-r^{2}\right)}{\left(1+r^{2}\right)^{N}+\left(1-r^{2}\right)^{N}}, \\
& \left\langle q_{1,2}^{2}\right\rangle_{+}\left(r^{2}\right)=\frac{\left(1+r^{2}\right)^{N}\left\langle q_{1,2}^{2}\right\rangle\left(r^{2}\right)+\left(1-r^{2}\right)^{N}\left\langle q_{1,2}^{2}\right\rangle\left(-r^{2}\right)}{\left(1+r^{2}\right)^{N}+\left(1-r^{2}\right)^{N}},
\end{aligned}
$$

for the symmetric one. Here $r$ is a shorthand for the equilibrium radius $r_{e}(\xi)$ and $r_{e}^{(N)}(\xi)$ in equations (37) and (38), respectively, so that fluctuations depend on $N$ and the control parameter $\xi$. Rotational symmetry implies $\left\langle q_{1}^{2}\right\rangle=\left\langle q_{2}^{2}\right\rangle$ in both cases. Note also that again, in both cases, the total variance:

$$
\left\langle q_{0}^{2}+q_{1}^{2}+q_{2}^{2}\right\rangle=\frac{3}{2}+N=\left\langle q_{0}^{2}+q_{1}^{2}+q_{2}^{2}\right\rangle_{+}
$$

is independent of $\xi$. Figures 8 and 9 compare fluctuations for variational symmetric $(N=2$ and $N=8)$ and non-symmetric $(N=\infty)$ ground state approximations (compare with the exact numerical values in Fig 2).

These pictures show that rescaled variances for the symmetric configuration are finite-size $(N<\infty)$ approximations of the variance for the non-symmetric configuration $(N=\infty)$ and tend to it in the thermodynamic limit. Therefore, whereas Rényi uncertainty is strongly sensitive to parity symmetry, variances are not. Finally, in figure 10 we have included a comparison between numerical, cat and mean-field variances for the case $N=20$ to appreciate the differences between them.

\section{SUMMARY}

We have studied Rényi entropies as a detector of the quantum phase transition in the vibron model. An ex- 


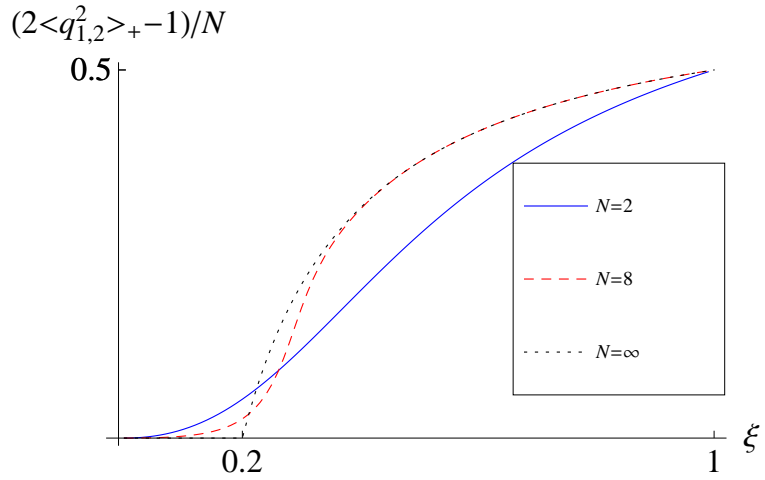

FIG. 9. (Color online) Normalized variance for the 'vector' components $\vec{q}=\left(q_{1}, q_{2}\right)$ in the cat $(N=2$ and 8$)$ and meanfiled $(N=\infty)$ approximations.

act diagonalization of the Hamiltonian shows that Rényi entropies have a sudden growth across the critical point. We also present a variational analysis in terms of CSs (mean-field configuration) and even-parity CSs (cat configuration) which enriches the analysis. Whereas Rényi entropies remain constant with the control parameter $\xi$ in the mean-field configuration, the cat configuration accounts for the QPT at the critical point $\xi_{c}$. We also compute variances for symmetric (cat) and nonsymmet- ric (mean-field) variational approximations. Variances for cat states match the exact results and both tend to variances of the mean-field in the limit $N \rightarrow \infty$. Variances are less sensitive to parity than Rényi entropies and scale with $N$ (they diverge in the thermodynamic limit), although they also provide a good description of the QPT. Rényi entropies are more sensitive in this particular model and it doesn't imply that it can be extrapolated to other physical systems, so we have to stress that, of course, variances continue being a good detector of the presence of a quantum phase transition. Finally, we have obtained some approximated analytical expressions for Rényi entropies in this model.

\section{ACKNOWLEDGMENTS}

This work was supported by the Projects: FIS201124149 and FIS2011-29813-C02-01 (Spanish MICINN), FQM-165/0207 and FQM219 (Junta de Andalucía) and 20F12.41 (CEI BioTic UGR). The work is also supported by the TAMOP 4.2.1/B-09/1/KONV-2010-0007 and the TAMOP 4.2.2/B-10/1-2010-0024 projects. The project is co-financed by the European Union and the European Social Fund. Grant OTKA No. K 100590 is also gratefully acknowledged.
[1] S. Sachdev, Quantum Phase Transitions, Cambridge University Press (2000).

[2] E. Romera and Á. Nagy, Phys. Lett. A 3753066 (2011).

[3] E. Romera, K. Sen and Á. Nagy, J.Stat. Mech. P09016 (2011).

[4] E. Romera, M. Calixto and Á. Nagy, Europhys. Lett. 97 (2012). doi:10.1209/0295-5075/97/2011.

[5] Á. Nagy and E. Romera, Physica A (2012),doi:10.1016/j.physa.2012.02.024

[6] A. Rényi, Proceedings of the 4th Berkeley Symposium on Mathematical Statistics and Probability, Volume 1: Contributions to the Theory of Statistics (1961) 547.

[7] R. H. Dicke, Phys. Rev. 93, 99 (1954).

[8] C. Emary and T. Brandes, Phys. Rev. E 67, 066203 (2003).

[9] C. Emary and T. Brandes, Phys. Rev. Lett. 90, 044101 (2003).

[10] C.E. Shannon, A mathematical theory of communication, Bell. Sys. Tech. J. 27 (1948) 379; ibid. (1948) 623.

[11] O. Gühne and M. Lewenstein, Phys. Rev. A 70, 022316 (2004).

[12] R. Renner, N. Gisin and B. Kraus, Phys. Rev. A 72 $012332(2005)$.

[13] P. Lévay, S. Nagy and J. Pipek, Phys. Rev. A 72, 022302 (2005).

[14] D. G. Arbo, C. O. Reinhold, J. Burgdörfer, A. K. Pattanayak, C. L. Stokely, W. Zhao, J. C. Lancaster and F. B. Dunning, Phys. Rev. A 67, 063401 (2003).
[15] E. Romera and F. de los Santos, Phys. Rev. A, 78, 013837 (2008).

[16] E. Romera and Á. Nagy, Phys. Lett. A 3724918 (2008).

[17] E.. Romera and Á. Nagy, Phys. Lett. A 372, 6823 (2008).

[18] Á. Nagy and E. Romera, Phys. Lett. A 373844 (2009).

[19] Á. Nagy and E. Romera, Int. J. Quantum Chem. 109 2490 (2009).

[20] M. Calixto, Á. Nagy, I. Paradela and E. Romera, Phys. Rev. A, In Press (2012).

[21] F. Iachello, Algebraic theory of Molecules, Oxford University Press (1995).

[22] F. Iachello, J. Chem. Phys. 78, 581 (1981).

[23] F. Iachello, S. Oss, J. Chem. Phys. 104, 6956 (1996).

[24] M. Calixto, E. Romera and R. del Real, J. Phys. A 45, 365301 (2012).

[25] F. Pérez-Bernal, F. Iachello, Phys. Rev. A 77, 032115 (2008).

[26] R. Gilmore, J. Math. Phys. 20, 891 (1979).

[27] A. Perelomov, Generalized Coherent States and Their Applications, Springer-Verlag (1986).

[28] J.R. Klauder, and Bo-Sture Skagerstam, Coherent States: Applications in Physics and Mathematical Physics, World Scientific Singapore (1985)

[29] S.T. Ali, J-P. Antoine, J.P. Gazeau, Coherent States, Wavelets and Their Generalizations, Springer (2000)

[30] A. Leviatan, M.W. Kirson, Ann. Phys. NY 188, 142 (1988)

[31] S. Kuyucak and M. K. Roberts, Phys. Rev A57, 3381 (1998) 

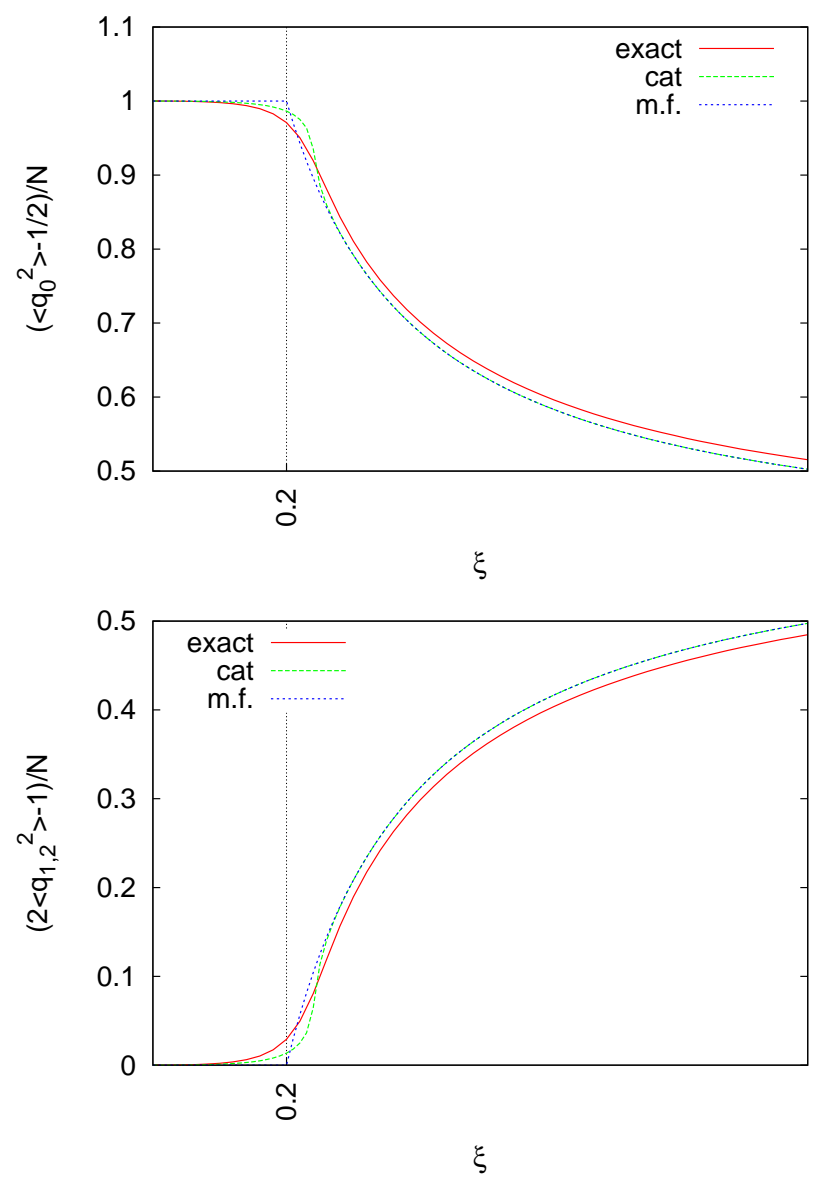

FIG. 10. (Color online) Numerical, cat and mean field normalized variances for the 'scalar' component $q_{0}$ and the 'vector' components $\vec{q}=\left(q_{1}, q_{2}\right)$ for $N=20$.
[32] M.A. Caprio, J. Phys. (Math. Gen.) A38, 6385 (2005)

[33] O. Castaños, E. Nahmad-Achar, R. López-Peña, J. G. Hirsch, Phys. Rev. A 83, 051601(R) (2011).

[34] O. Castaños, E. Nahmad-Achar, R. López-Peña, J. G. Hirsch, Phys. Rev. A 84, 013819 (2011).

[35] P. Pérez-Fernández, J. M. Arias, J. E. García-Ramos, and F. Pérez-Bernal, Phys. Rev. A 83, 062125 (2011).

[36] S. Dusuel, J. Vidal, J. M. Arias, J. Dukelsky and J. E. García-Ramos, Phys. Rev. C 72, 064332 (2005).

[37] W. Van Assche, R. J. Yánez and J. S. Dehesa, J. Math. Phys. 36, 4106 (1995).

[38] P. Sánchez-Moreno, J. S. Dehesa, D. Manzano and R. J. Yánez, J. Comp. Appl. Math. 233, 2136 (2010).

[39] A. I. Aptekarev, V. S. Buyarov, W. Van Assche and J. S. Dehesa, Doklady Math. 53, 47 (1996).

[40] A. I. Aptekarev, V. S. Buyarov and J. S. Dehesa, Russian Acad. Sci. Sb. Math. 82, 373 (1995).

[41] J. S. Dehesa, W. Van Assche and R. J. Yánez, Phys. Rev. A 50, 3065 (1994).

[42] J. S. Dehesa, W. Van Assche and R. J. Yánez, J. Appl. Anal. 4, 91 (1997).

[43] A. I. Aptekarev, J. S. Dehesa, A. Martinez-Finkelstein, J. Comp. Appl. Math. 233, 1335 (2010). 